\title{
Ecological theory of mutualism: Robust patterns of stability and thresholds in two-species population models
}

\author{
Kayla Hale ${ }^{1}$ and Fernanda Valdovinos ${ }^{2}$ \\ ${ }^{1}$ University of Michigan \\ ${ }^{2}$ University of California Davis
}

November 29, 2021

\begin{abstract}
Mutualisms are ubiquitous in nature, provide important ecosystem services, and involve many species of interest for conservation. Theoretical progress on the population dynamics of mutualistic interactions, however, comparatively lagged behind that of trophic and competitive interactions, leading to the impression that ecologists still lack a generalized framework to investigate the population dynamics of mutualisms. Yet, over the last 90 years, abundant theoretical work has accumulated, ranging from abstract to detailed. Here, we review and synthesize historical models of two-species mutualisms. We find that population dynamics of mutualisms are qualitatively robust across derivations, including levels of detail, types of benefit, and inspiring systems. Specifically, mutualisms tend to exhibit stable coexistence at high density and destabilizing thresholds at low density. These dynamics emerge when benefits of mutualism saturate, whether due to intrinsic or extrinsic density-dependence in intraspecific processes, interspecific processes, or both. We distinguish between thresholds resulting from Allee effects, low partner density, and high partner density, and their mathematical and conceptual causes. Our synthesis suggests that there exists a robust population dynamic theory of mutualism that can make general predictions.
\end{abstract}

\section{Hosted file}

Hale \& Valdovinos 2021 Ecological theory of mutualism- Qualitative patterns in two-species population m available at https://authorea.com/users/418871/articles/547219-ecological-theory-ofmutualism-robust-patterns-of-stability-and-thresholds-in-two-species-population-models 